\title{
Time-dependent MHD shocks and line emission: the case of the DG Tau jet
}

\author{
S. Massaglia ${ }^{1}$, A. Mignone ${ }^{2}$, and G. Bodo ${ }^{2}$ \\ 1 Dipartimento di Fisica Generale dell'Università, via Pietro Giuria 1, 10125 Torino, Italy \\ e-mail: massaglia@ph.unito.it \\ 2 Osservatorio Astronomico di Torino, Viale Osservatorio 20, 10025 Pino Torinese, Italy \\ e-mail: [mignone; bodo]@to.astro.it
}

Received 2 May 2005 / Accepted 21 July 2005

\section{ABSTRACT}

\begin{abstract}
The line emission from a growing number of Herbig-Haro jets can be observed and resolved at angular distances smaller than a few arcseconds from the central source. The interpretation of this emission is problematic, since the simplest model of a cooling jet cannot sustain it. It has been suggested that what one actually observes are shocked regions with a filling factor of $\sim 1 \%$. In this framework, up to now, comparisons with observations have been based on stationary shock models. Here we introduce for the first time the self-consistent dynamics of such shocks and we show that considering their properties at different times, i.e. locations, we can reproduce observational data of the DG Tau microjet. In particular, we can interpret the spatial behavior of the [SII]6716/6731 and [NII]/[OI]6583/6300 line intensity ratios adopting a set of physical parameters that yield values of mass loss rates and magnetic fields consistent with previous estimates. We also obtain the values of the mean ionization fraction and electron density along the jet and compare these values with those derived from observations using the sulfur doublet to constrain the electron density.
\end{abstract}

Key words. ISM: jets and outflows - magnetohydrodynamics (MHD) - hydrodynamics - stars: early-type

\section{Introduction}

Herbig-Haro jets can be observed at very high spatial resolution by HST and with the next generation of ground based optical telescopes such as VLTI/AMBER the angular resolution capabilities will be boosted from the actual fraction of an arcsecond up to the milliarcsecond range (Bacciotti 2004). It will soon be possible to look into the very first part of the jet as it emerges from the accretion disk or from the reflection nebula and resolve many jets in their radial extent. It will thus be feasible to perform a comparison of direct observations with the predictions of the acceleration models. Some jets (sometimes called "microjets"), e.g. HH 30 (Bacciotti et al. 1999) or DG Tau (Lavalley et al. 1997; Lavalley-Fouquet et al. 2000 (L-FCD2000); Bacciotti et al. 2000), are particularly good candidates for a high resolution study of the evolution of the physical parameters along the initial fraction of the jet, i.e. up to $\sim 10^{16} \mathrm{~cm}$ where the conditions to meet for the line emission mechanisms at work are the most severe.

The jet of DG Tau has been observed close to its base at CFHT at high angular resolution $\left(\sim 0{ }^{\prime} 5\right)$ by L-FCD2000 who showed the behavior of [SII]6716/6731 and $[\mathrm{NII}] /[\mathrm{OI}] 6583 / 6300$ line ratios along the jet for the high (HV), intermediate (IV) and low (LV) velocity components. Our calculations will address these data in modeling the jet line emission.

These observations typically show that the behavior of temperature, ionization and density along the jet is incompatible with a freely cooling jet. Various heating processes have been proposed in the literature, such as ambipolar diffusion (Garcia et al. 2001a,b), photoionization by soft X-rays from the TTauri star (e.g. Shang et al. 2002) and mechanical heating (O'Brien et al. 2003; Shang et al. 2002, for X-wind jets). These estimates where carried out for steady-state jet models and pointed out that mechanical heating was the most effective in reproducing the observations. The idea of tapping a small fraction of the jets kinetic energy to convert into heat is certainly appealing; however there is no physical explanation for if and how this process could work in YSO jets. Velocity fluctuations may possibly steepen into shocks and dissipate their energy heating the gas, but that radiative losses come into play and act against the heating process.

An alternative explanation by L-FCD2000 to interpret the line ratios of DG Tau (see also Hartigan 2004), is that one observes several post-shock regions of high excitation with a filling factor of $\sim 1 \%$ ("shocking jet"). L-FCD2000 found that the line ratios of DG Tau and other HH objects (compilation by Raga et al. 1996) might be interpreted as series of 
shocks arrayed along the jet with varying shock velocities as one moves along the radius away from the jet axis, typically: $70-100 \mathrm{~km} \mathrm{~s}^{-1}$ for the HV component, $50-60 \mathrm{~km} \mathrm{~s}^{-1}$ for IV and pre-shock densities that decrease with the distance from the star as $\propto r^{-2}$ starting from $n_{0}=10^{5} \mathrm{~cm}^{-3}$. In their analysis, they considered post-shock parameters consistent with planar, stationary shocks models (e.g. Hartigan et al. 1994).

Raga et al. (2001) modeled the morphological and dynamical properties of the DG Tau jet by means of 3D numerical simulations, assuming a precessing jet with a velocity that varied sinusoidally in time. In the present paper we restrict ourselves to the interpretation of the line intensity ratio behavior along the jet, abandoning the assumption of stationary shocks. We follow the dynamical evolution of an initial perturbation as it steepens into a (radiative) shock traveling along the jet, and derive the post-shock physical parameters consistently (Massaglia et al. 2005a). From these parameters we construct the emission line ratios to be compared with observations. In this first analysis we consider the evolution of a single shock, neglecting the possible interaction with other shocks.

The plan of the paper is the following: in Sect. 2 we outline the initial conditions and the computational scheme adopted; in Sect. 3 we examine the shock evolution, while in Sect. 4 we discuss the results and make comparisons with observations. Our conclusions are drawn in Sect. 5.

\section{The model}

\subsection{Basic equations}

We restrict our attention to one-dimensional MHD planar flow. The fluid is described in terms of its density $\rho$, velocity $u$, thermal pressure $p$ and (transverse) magnetic field $B_{y}$. Conservation of mass, momentum, magnetic field and total energy readily follows:

$\frac{\partial \rho}{\partial t}+\frac{\partial(\rho u)}{\partial x}=0$

$\frac{\partial(\rho u)}{\partial t}+\frac{\partial}{\partial x}\left(\rho u^{2}+p+\frac{B_{y}^{2}}{2}\right)=0$,

$\frac{\partial B_{y}}{\partial t}+\frac{\partial\left(B_{y} u\right)}{\partial x}=0$

$\frac{\partial E}{\partial t}+\frac{\partial}{\partial x}\left[\left(E+p+\frac{B_{y}^{2}}{2}\right) u\right]=-\mathcal{L}\left(T, f_{\mathrm{n}}\right)$,

where $E=p /(\Gamma-1)+\rho u^{2} / 2+B_{y}^{2} / 2$ is the total energy density (we use $\Gamma=5 / 3$ ) and $\mathcal{L}\left(T, f_{\mathrm{n}}\right)$ represents the energy loss term (energy per unit volume per unit time) which depends on the temperature $T$ and, as explained below, on the number fraction of neutral hydrogen atoms, $f_{\mathrm{n}}$. The loss term accounts for energy lost in lines and in the ionization and recombination processes. Line emissions include contributions from nine elements whose abundances have been assumed to be solar (Anders \& Grevesse 1989): $\mathrm{H}$ and He resonance lines, the 13 strongest forbidden lines of $\mathrm{C}, \mathrm{N}, \mathrm{O}, \mathrm{S}, \mathrm{Si}, \mathrm{Fe}$ and $\mathrm{Mg}$. He is neutral whereas metals are singly ionized and the abundance of $\mathrm{C}$ is $10 \%$ of the solar one. The ionization states of $\mathrm{N}$ and $\mathrm{O}$ are fixed to that of $\mathrm{H}$ by charge transfer.

To compare between the observed and computed line ratios, we have computed the populations of the atomic levels for the forbidden transitions [SII] $\lambda \lambda 6716,6731,[\mathrm{NII}] \lambda 6583$ and [OI] $\lambda 6300$, solving, according to Osterbrock (1974), the excitation - de-excitation equilibrium equations for five energy levels.

An additional evolutionary equation is solved for the neutral fraction $f_{\mathrm{n}}$,

$\frac{\partial f_{\mathrm{n}}}{\partial t}+v \frac{\partial f_{\mathrm{n}}}{\partial x}=n_{\mathrm{e}}\left[-c_{\mathrm{i}} f_{\mathrm{n}}+c_{\mathrm{r}}\left(1-f_{\mathrm{n}}\right)\right]$

where $n_{\mathrm{e}}$ is the electron density, whereas $c_{\mathrm{i}}$ and $c_{\mathrm{r}}$ are, respectively, the ionization and recombination rate coefficients (Rossi et al. 1997). In this framework we have

$n_{\mathrm{e}}=n_{\mathrm{H}}\left(1-f_{\mathrm{n}}\right)+Z n_{\mathrm{H}}$,

and

$p=n_{\mathrm{H}}\left(2-f_{\mathrm{n}}+Y+2 Z\right) k_{\mathrm{B}} T$,

where $n_{\mathrm{H}}$ is the total hydrogen density, $Y$ and $Z(=0.001)$ are the helium and metal abundances by number respectively. We will also define the ionization fraction as

$f_{\mathrm{i}}=\frac{n_{\mathrm{e}}}{n_{\mathrm{H}}}$.

\subsection{The initial perturbation}

A nonuniform pre-shock density that decreases away from the star is a reasonable ingredient when dealing with an expanding jet, as suggested by observations. Moreover, shocks that propagate into a stratified medium tend to increase their amplitude when they find a decreasing pre-shock density. Therefore, we consider the following nonuniform pre-shock density:

$\rho_{0}(x)=\rho_{0} \frac{x_{0}^{2}}{x_{0}^{2}+x^{2}}$,

where $x$ is the spatial coordinate along the jet axis. Thus, for $x \gg x_{0}$, we have a conical decrease of density, i.e. a conical expansion of the jet, while for $x \lesssim x_{0}$ the density decreases parabolically, $\rho_{0}(x) \approx \rho_{0}\left(1-x^{2} / x_{0}^{2}\right)$. $x_{0}$ sets the initial steepness of the density function and this affects the shock evolution even at larger distances.

A generic initial perturbation imposed above the mean flow typically evolves forming two shocks: the forward and reverse shocks (Hartigan \& Raymond 1993; Massaglia et al. 2005a). The energy content of the perturbation therefore splits into two radiating shocks that propagate, decreasing their strengths along the way. Thus one cannot reach and maintain the compression factors to explain observations, as happened in Massaglia et al. (2005a), unless assuming an initial perturbation velocity amplitude of the order of the jet mean flow speed. Thus it would be more desirable to work with a single forward shock. To this purpose, we consider a disturbance that maintains the Riemann invariant $J_{-}$constant (Zeldovich \& Raizer 1966):

$J_{-}=u-\int \frac{\mathrm{d} p}{\rho c}=u-\int \frac{\mathrm{d} p}{\mathrm{~d} u} \frac{\mathrm{d} u}{\rho c}=$ const. 

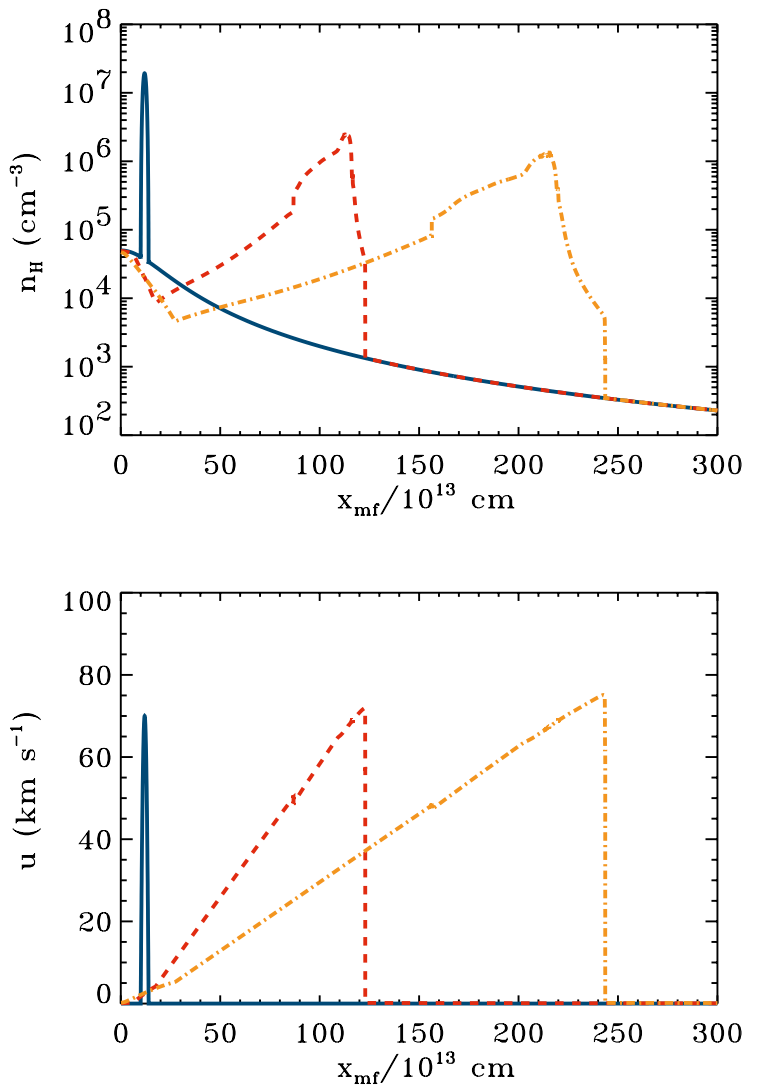

Fig. 1. Density (upper panel) and velocity (lower panel) vs. distance in the reference frame at rest with the mean flow (abscissa $x_{\mathrm{mf}}$ ); solid lines show the initial perturbation, the dashed lines the evolution at $t=5 \mathrm{ys}$ and dot-dashed lines at $t=10 \mathrm{ys}$.

After differentiation and some algebra, one finds

$\rho=\left[\frac{\gamma-1}{2 \sqrt{K \gamma}}\left(u-U_{0}\right)+\rho_{0}^{\frac{\gamma-1}{2}}\right]^{\frac{2}{\gamma-1}}$.

We set $U_{0}(x)=0$, implying that we carry out our calculations in the reference frame of the mean flow. We will have to transform our data to the laboratory frame when comparing results with observations. We prescribe the velocity perturbation as

$u(x)= \begin{cases}u_{0}\left[-\left(x-x_{1}\right)^{2}+2 \sigma\left(x-x_{1}\right)\right] & \text { if } 2 \sigma+x_{1}>x>x_{1} \\ 0 & \text { otherwise }\end{cases}$

where $x_{1}$ is the initial coordinate of the perturbation and $\sigma$ is its half-width (see Fig. 1, solid line). Different choices of the initial perturbation shape are not crucial for the shock formation and evolution.

Notice that, strictly speaking, $J_{-}$in Eq. (10) is no longer an hydrodynamic invariant when magnetic fields are present. In our case, however, the initial magnetic pressure is typically small and the resulting perturbation still produces a strong forward shock, as shown in Fig. 1. In our calculations we have assumed $x_{1}=10^{14} \mathrm{~cm}$ and $\sigma=2 \times 10^{13} \mathrm{~cm}$.

The boundary conditions assume free outflow at $x=0$ and $x=L$. The extent of the computational domain, $L$, has been chosen sufficiently large to follow the shock evolution for $t \sim$ 15 ys and avoid spurious interactions with the boundaries. For this reason we adopt $L=4.5 \times 10^{15} \mathrm{~cm}$.

\subsection{Method of solution}

To solve the MHD equations we have employed the hydrodynamical code PLUTO (Mignone et al. 2004).

Equations (1)-(4), together with Eq. (5) are solved using a conservative, second-order accurate total variation diminishing (TVD) scheme. Piecewise linear interpolation with limited slopes is used to ensure monotonicity inside each computational zone. Second order accuracy in time is achieved via a MUSCL-Hancock predictor step (van Leer 1985). A linear, Roe-type, Riemann solver is used to compute the inter-cell fluxes needed in the conservative update. The conservative formulation is essential for a correct description of the shock dynamics (LeVeque et al. 1998). Source terms describing cooling, ionization and recombination processes are treated using operator splitting.

In the simulations presented below, the region of interest is confined mainly to the post-shock flow, where most of the emission takes place. Since this region is much smaller in size $\left(\lesssim 7 \times 10^{13} \mathrm{~cm}\right)$ than the domain and the shock front is not stationary, a static uniform grid would demand increasingly high resolution in order to adequately resolve the post-shock structure. For this reason, we use an Adaptive Mesh Refinement (AMR) technique (described in Massaglia et al. 2005b) when solving Eqs. (1)-(4), thus reaching an effective resolution of about 360000 grid points.

This allows to considerably speed up the computation with sufficient resolution to accurately describe the dynamics and emission processes.

\section{Results and discussion}

We have followed the temporal evolution of the perturbation as it steepened into a shock traveling along the computational domain (see Fig. 1). We have been able, with the AMR technique employed, to resolve the post-shock region at all times and in Fig. 2 we show the post-shock variables versus the spatial coordinate. Our model calculations depend on the following parameters: the initial perturbation velocity amplitude $u_{0}$, the preshock magnetic field transverse to the flow $B_{0}$, the pre-shock density parameters $x_{0}$ and $n_{0}$ and the mean flow speed $U_{0}$, to transform the results back to the observer's reference frame. Test calculations have shown that, among these parameters, $u_{0}$ and the pre-shock density parameters $x_{0}$ and $n_{0}$ are the most critical for the final results. We have fixed the (isothermal) preshock temperature $T=1000 \mathrm{~K}$ (Raga et al. 2001) and ionization fraction $f_{\mathrm{i}}=Z$ due to metals only. However results are weakly sensitive to these values, for the range of parameters considered. In Fig. 2 we plot the post-shock temperature $T$, electron density $\left(n_{\mathrm{e}}\right)$, ionization fraction $\left(f_{\mathrm{i}}\right)$, magnetic field $B_{y}$ in units of $B_{0}$, [SII] emissivity (in units of the maximum value, rightmost vertical scale) and velocity $u$, in the reference frame of the mean flow. The horizontal scale has an arbitrary origin and tells us the size of the post-shock region. The values of the parameters are: $u_{0}=70 \mathrm{~km} \mathrm{~s}^{-1}, B_{0}=100 \mu \mathrm{G}, x_{0}=0 . ' 1$ and $n_{0}=5 \times 10^{4} \mathrm{~cm}^{-3}$.

The post-shock quantities are shown after $10 \mathrm{ys}$ of evolution, when the shock front has traveled for about $6.5 \times 10^{15} \mathrm{~cm}$ 


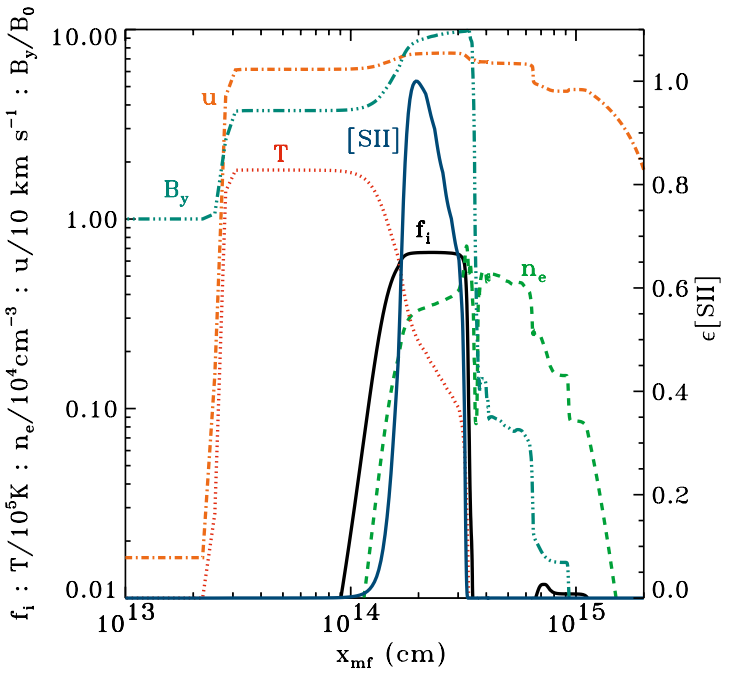

Fig. 2. Spatial behavior, in the mean flow frame, of the physical quantities as they undergo the shock transition, from left to right, after $10 \mathrm{ys}$ of evolution, corresponding to a traveled distance of the shock front of about $6.5 \times 10^{15} \mathrm{~cm}$ along the jet axis. Labels are: $f_{\mathrm{i}}$ ionization fraction (solid line), $n_{\mathrm{e}}$ electron density (dashed line), $T$ temperature (dotted line), $u$ velocity (dot-dashed line), $B_{y}$ magnetic field (in units of the pre-shock value $B_{0}$, dot-dot-dashed line) and [SII] emissivity (solid line).

from the source, having assumed a mean flow speed $U_{0}=$ $150 \mathrm{~km} \mathrm{~s}^{-1}$ (see below). We note that the post-shock temperature attains a value $\gtrsim 10^{5} \mathrm{~K}$ right behind the shock and decreases to below $10^{4} \mathrm{~K}$ about $7 \times 10^{13} \mathrm{~cm}$ from the shock front, where the [SII] emission takes place and the magnetic field reaches its maximum and subsequently drops to low values at $\sim 10^{14} \mathrm{~cm}$. We see that both the (collisional) ionization fraction and the electron density reach maximum values at about $7 \times 10^{13} \mathrm{~cm}$ behind the shock front. In the post-shock region the particle velocity remains nearly constant to the original value of the perturbation amplitude and decreases starting at $\sim 2 \times 10^{15} \mathrm{~cm}$ from the shock front. We are not plotting the post-shock speed in the frame where the shock is at rest. At earlier times, the general behavior would be almost unchanged for each quantity exception made for the electron density which would present a maximum that is higher by about a factor of three due to the higher (imposed) pre-shock density. Clearly, the opposite happens at later times in the evolution.

In order to carry out a comparison with observations we need to average the post-shock quantities at every evolutionary time point. We have done this following Hartigan et al. (1994), i.e. defining the $[\mathrm{SII}]$-weighted average as follows

$\langle Q\rangle=\frac{\int Q(x) \epsilon\{[\mathrm{SII}](x)\} \mathrm{d} x}{\int \epsilon\{[\mathrm{SII}](x)\} \mathrm{d} x}$

where $Q$ is a physical quantity such as either electron density or ionization fraction or the line emissivity ratio of the sulfur doublet [SII] $\lambda 6716 /[$ SII] $\lambda 6731$. Concerning the line emissivity ratio $[\mathrm{NII}] \lambda 6583 /[\mathrm{OI}] \lambda 6300$, we have carried out the averaging procedure in the same way, but adopting the total emissivity $\epsilon\{[\mathrm{NII}](x)\}+\epsilon\{[\mathrm{OI}](x)\}$ as the weighting function. However, we noticed that the particular choice of the weighting function

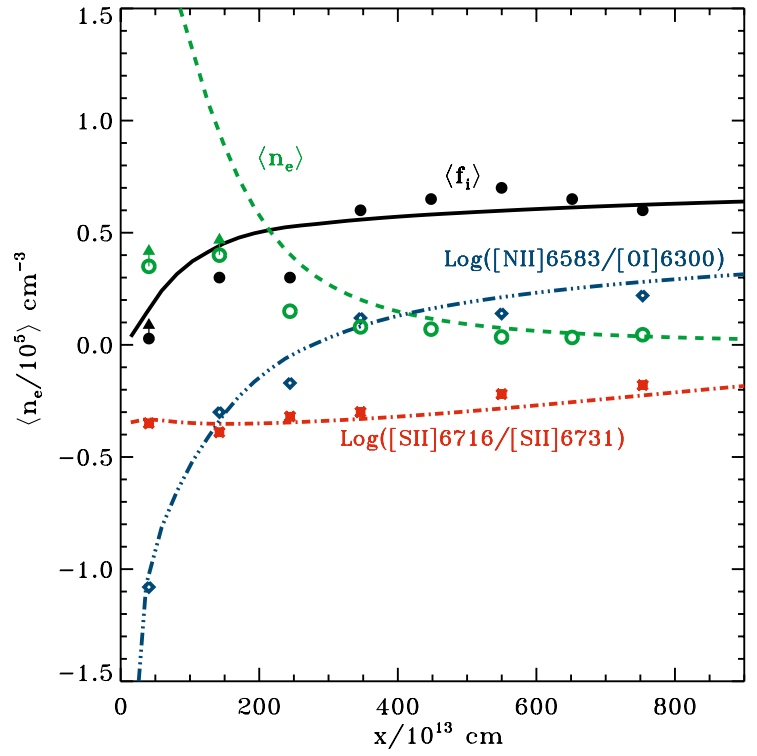

Fig. 3. Averaged values of the post-shock electron density $\left(\left\langle n_{\mathrm{e}}\right\rangle\right.$, dashed line), ionization fraction $\left(\left\langle f_{\mathrm{i}}\right\rangle\right.$ solid line), ratios of [SII] (dotdashed line) and $[\mathrm{NII}] /[\mathrm{OI}]$ (dot-dot-dashed line) along the jet for the reference parameters vs distance (in the laboratory frame): $u_{0}=$ $70 \mathrm{~km} \mathrm{~s}^{-1}, B_{0}=100 \mu \mathrm{G}, x_{0}=00^{\prime} 1$ and $n_{0}=5 \times 10^{4} \mathrm{~cm}^{-3}$ (see also Fig. 2). Here we have set $U_{0}=150 \mathrm{~km} \mathrm{~s}^{-1}$.

has very little effect on the results. Note also from Fig. 2 that for $f_{\mathrm{i}}$ and $B_{y}$ the averaging procedure will yield, with a good approximation, the maximum value of these quantities.

In Fig. 3 we compare of the averaged post-shock quantities, obtained for the above set of parameters and assuming a mean outflow velocity $U_{0}=150 \mathrm{~km} \mathrm{~s}^{-1}$, with the observations by L-FCD2000. The abscissa indicates the position of the post-shock region in the observer's frame, $x=U_{0} t+\xi(t)$, where $t$ is the elapsed time and $\xi(t)$ is the location of the emission region. We have chosen the high velocity (HV) jet data of the line ratios, ionization fraction and electron density from the paper of L-FCD2000, likely corresponding to the inner part of the jet about its longitudinal axis. After examining Fig. 2, we have estimated $\xi(t)$ as the shock front position minus an interval $d\left(=2 \times 10^{14} \mathrm{~cm}\right)$ that accounts for the shift of the emission region behind the shock front. The results are nearly insensitive to the choice of $d$. We recall that the directly observable data are the line intensity ratios (in Fig. 3, crosses are for the $[\mathrm{SII}]$ doublet and diamonds for the $[\mathrm{NII}] /[\mathrm{OI}]$ ratio), while the ionization fraction and electron density have been obtained by L-FCD2000 adopting the diagnostic technique described in Bacciotti et al. (1995). From Fig. 3 we note that the $[\mathrm{NII}] /[\mathrm{OI}]$ and $[\mathrm{SII}]$ ratios fit the data remarkably well, while the electron density and the ionization fraction exceed the ones derived from observations at low values of the spatial coordinate $x$. In fact, Bacciotti et al. (1995) obtain $n_{\mathrm{e}}$ from the sulfur doublet line intensity ratio, which saturates for electron densities higher than the critical density $n_{\mathrm{c}} \lesssim 10^{4} \mathrm{~cm}^{-3}$. This leads to an underestimation of the electron density and, consequently, the ionization fraction. Note that in L-FCD2000 the first couple of data points of the electron density and the first one of the ionization fraction are lower limits. Instead, this method is 
Table 1. Behavior of the line ratio curves with the increase of the parameter in the leftmost column.

\begin{tabular}{ccc}
\hline \hline Parameter & {$[\mathrm{NII}] 6583 /[\mathrm{OI}] 6300$} & {$[\mathrm{SII}] 6716 /[\mathrm{SII}] 6731$} \\
\hline$n_{0}$ & $\downarrow$ & $\downarrow$ \\
$u_{0}$ & $\uparrow$ & $\downarrow$ \\
$x_{0}$ & $\downarrow$ & $\downarrow$ \\
$B_{0}$ & $\uparrow$ & $\uparrow$ \\
\hline
\end{tabular}

appropriate for lower values of the electron density, thus the agreement in Fig. 3 at larger distances.

Several questions arise: which sets of parameters better represent the observations? What are the crucial parameters?

Variations of the advection velocity $U_{0}$ have no physical impact on the shock evolution but concern only a dilation of $x$ axis. We varied $U_{0}$ by $\pm 40 \%$ and noted that the agreement remained reasonably good. Thus values of $U_{0}$ within these two limits still interpret observations reasonably well.

We are left with four main parameters: $n_{0}, x_{0}, B_{0}$ and $u_{0}$. Due to the difficulty in carrying out a full exploration of the parameter space, we have synthesized the "goodness" of the agreement between model curve and observational points of the line ratios defining a variance $\sigma^{2}=\sum_{i}\left(O_{i}-E_{i}\right)^{2}$, where $O_{i}$ are the data and $E_{i}$ the model points. In Table 1 we show the qualitative behavior of the $[\mathrm{SII}]$ and $[\mathrm{NII}] /[\mathrm{OI}]$ ratio model curves when a parameter on the left column is increased, leaving the other ones unchanged with their reference values of Fig. 3: these curves are displaced downward when $n_{0}$ and $x_{0}$ are raised, changing in shape as well, while the curves shift upward when raising $B_{0}$. We have run several models (according to Table 1 ) varying the parameters $n_{0}, B_{0}, u_{0}$ and $x_{0}$ around the reference set and calculated the variance $\sigma^{2}$ of the corresponding fits. Since for most of the spatial coordinate $x$ we are above the critical density, the variance of the fits of the [SII] doublet line ratio is not very sensitive to the changes in these parameters. However, this is not the case for the $[\mathrm{NII}] /[\mathrm{OI}]$ curves: we could obtain fits for $[\mathrm{NII}] /[\mathrm{OI}]$ with a variance close to the minimum by varying $n_{0}, x_{0}$ and $B_{0}$ by about $30 \%$ and $u_{0}$ by about $10 \%$ plus or minus with respect to the reference values.

As far as the magnetic field evolution is concerned, direct comparisons with previous 2D models (e.g., Garcia et al. 2001a) and 3D simulations (e.g., Cerqueira \& de Gouveia dal Pino 2001) are difficult because we only include the transverse component of the magnetic field. The spatial evolution of the mean field in our models typically shows a monotonic decrease from about $30 B_{0}$ down to about $5 B_{0}$. If we consider the behavior of $B_{\phi}$ from Garcia et al. (2001a), our values of the magnetic field, with $B_{0}=100 \mu \mathrm{G}$, remain a factor of $\sim 2-3$ below the curve of their model $\mathrm{C}$.

\section{Conclusions}

With the goal to interpret the line-emission within the first $5^{\prime \prime}$ observed in some Herbig-Haro jets, we have considered the evolution of a strong planar perturbation in a stratified, magnetized medium and in the presence of radiative losses. We have examined the temporal evolution of this perturbation as it steepened into a shock and focused our attention to the structure of the post-shock region. Having set physical parameters consistent with the environment of stellar jets in their inner portions, closer to the young star, we have derived averaged line intensity ratios that could be compared with observations. We have adopted observations of line ratios of the DG Tau jet by L-FCD2000, as an example, and found that even in this extremely simple model, one can interpret observations with a constrained set of parameters reasonably well.

In this model we have a priori prescribed the longitudinal density profile upstream of the perturbation, assuming that a real jet, in the same physical conditions, would expand decreasing its density along the way. Whether an actual jet behaves in a similar fashion remains to be seen and, to this purpose, 2D MHD simulations of radiative jets are needed. The reference set of parameters that yield a "good" agreement with observations are: $u_{0}=70 \mathrm{~km} \mathrm{~s}^{-1}, B_{0}=100 \mu \mathrm{G}$, $x_{0}=0^{\prime} 1, n_{0}=5 \times 10^{4} \mathrm{~cm}^{-3}$ with a mean flow speed $U_{0}=150 \mathrm{~km} \mathrm{~s}^{-1}$. Assuming an initial radius of $3 \times 10^{14} \mathrm{~cm}$ (Raga et al. 2001), these values are consistent with a mass loss rate $\dot{M} \approx 5 \times 10^{-8} M_{\odot} \mathrm{ys}^{-1}$, in agreement with the estimates of L-FCD2000. The presence of a perturbation raises this value to $\dot{M} \approx 10^{-6} M_{\odot} \mathrm{ys}^{-1}$ for short periods.

One might ask whether these shocks may also be responsible for the emission knots observed in some $\mathrm{HH}$ jets (e.g. $\mathrm{HH} 34, \mathrm{HH} 111)$ at larger distances from the source $\left(\$ 45^{\prime \prime}\right)$ (cf. a review by Raga et al. 2004; Micono et al. 1998a,b). Due to the strong emissivity of the shock compressed medium, we believe that these shocks would hardly be visible at very large distances, as observed.

Thus, these calculations successfully reproduce line-ratio observations and thus strongly support the hypothesis of L-FCD2000 of the DG Tau jet as a "shocking jet", i.e. that one actually observes not a continuous emitting jet but just the gas parcels that have undergone compression, heating and ionization in shocks.

Acknowledgements. We thank Sylvie Cabrit for stimulating suggestions and helpful comments. We are also grateful to Pat Hartigan for discussions and to Paulo Garcia who, as referee, helped us to improve the paper. We acknowledge the Italian MIUR for financial support, grants No. 2002.028843 and No. 2004.025227. The present work was supported in part by the European Community Marie Curie Actions - Human resource and mobility within the JETSET network under contract MRTN-CT-2004 005592.

\section{References}

Anders, E., \& Grevesse, N. 1989, Geoc. Cosm. Acta, 53, 197 Bacciotti, F., Chiuderi, C., \& Oliva, E., 1995, A\&A, 296, 185

Bacciotti, F., Eislöffel, J., \& Ray, T. P. 1999, A\&A, 350, 917

Bacciotti, F., Mundt, R., Ray, T. P., et al. 2000, ApJ, 537, L49

Bacciotti, F. 2004, Ap\&SS, 293, 37

Cerqueira, A. H., \& de Gouveia dal Pino, E. M. 2001, ApJ, 550, L91

Garcia, P. J. V., Ferreira, J., Cabrit, S., \& Binette, L. 2001a, A\&A, 377, 589

Garcia, P. J. V., Cabrit, S., Ferreira, J., \& Binette, L. 2001b, A\&A, 377, 609

Hartigan, P., \& Raymond, J. 1993, ApJ, 409, 705 
Hartigan, P., Morse, J. A., \& Raymond, J. 1994, ApJ, 436, 125

Hartigan, P. 2004, Paper presented at the Banff Conference on: Cores, Disks, Jets \& Outflows in Low \& High Mass Stars Forming Environments, July 2004

Lavalley, C., Cabrit, S., \& Dougados, C. 1997, A\&A, 337, 671

Lavalley-Fouquet, C., Cabrit, S., \& Dougados, C. 2000, A\&A, 356, L41 (L-FCD2000)

LeVeque, R. J., Mihalas, D., Dorfi, E. A., \& Müller, E. 1998, Computational Methods for Astrophysical Flow (SpringerVerlag)

Massaglia, S., Mignone, A., \& Bodo, G. 2005a, Mem. S. A. It., 76, 378

Massaglia, S., Mignone, A., Bodo, G., \& Bacciotti, F. 2005b, in preparation

Micono, M., Massaglia, S., Bodo, G., Rossi, P., \& Ferrari, A. 1998a, A\&A, 333, 989
Micono, M., Massaglia, S., Bodo, G., Rossi, P., \& Ferrari, A. 1998b, A\&A, 333, 1001

Mignone, A., Massaglia, S., \& Bodo, G. 2004, Ap\&SS, 293, 199

O’Brien, D., \& Garcia, P. 2003, Ap\&SS, 287, 3

Raga, A., Böhm, K. H., \& Canto, J., Rev. Mex. Astron. Astrofis., 32, 161

Raga, A. C., Beck, T., \& Riera, A. 2004, Ap\&SS, 293, 27

Raga, A. C., Cabrit, S., Dougados, C., \& Lavalley, C. 2001, A\&A, 367,959

Rossi, P., Bodo, G., Massaglia, S., \& Ferrari, A. 1997, A\&A, 321, 672

Shang, H., Glassgold, A. E., Shu, F. H., \& Lizano, S. 2002, ApJ, 564, 853

van Leer, B. 1985, SIAM J. Sci. Stat. Comput., 5, 1

Zeldovich, Ya. B., \& Raizer, Yu. P., 1966, in Physics of Shock Waves and High-Temperature Hydrodynamic Phenomena (Academic Press), 1, 27 